\title{
1 Content-Aware Image Restoration: Pushing the Limits of 2 Fluorescence Microscopy
}

3 Martin Weigert ${ }^{1,2, *}$, Uwe Schmidt ${ }^{1,2}$, Tobias Boothe ${ }^{2}$, Andreas Müller ${ }^{8,9,10}$, Alexandr

4 Dibrov $^{1,2}$, Akanksha Jain ${ }^{2}$, Benjamin Wilhelm ${ }^{1,6}$, Deborah Schmidt ${ }^{1}$, Coleman

5 Broaddus $^{1,2}$, Siân Culley ${ }^{4,5}$, Mauricio Rocha-Martins ${ }^{1,2}$, Fabián Segovia-Miranda ${ }^{2}$, Caren

6 Norden $^{2}$, Ricardo Henriques ${ }^{4,5}$, Marino Zerial ${ }^{1,2}$, Michele Solimena ${ }^{2,8,9,10}$, Jochen Rink ${ }^{2}$,

7 Pavel Tomancak ${ }^{2}$, Loic Royer ${ }^{1,2,7, *}$, Florian Jug ${ }^{1,2, *, \dagger}$ \& Eugene W. Myers ${ }^{1,2,3, \dagger}$

$8 \quad{ }^{1}$ Center for Systems Biology Dresden (CSBD), Dresden, Germany

$9 \quad{ }^{2}$ Max-Planck Institute of Molecular Cell Biology and Genetics, Dresden, Germany

$10 \quad{ }^{3}$ Department of Computer Science, Technical University Dresden

${ }^{4}$ MRC Laboratory for Molecular Cell Biology, University College London, London, UK

${ }^{5}$ The Francis Crick Institute, London, UK

${ }^{6}$ University of Konstanz, Konstanz, Germany

${ }^{7}$ CZ Biohub, San Francisco, USA

${ }^{8}$ Molecular Diabetology, University Hospital and Faculty of Medicine Carl Gustav Carus, TU Dresden, Dresden, Germany

${ }^{9}$ Paul Langerhans Institute Dresden (PLID) of the Helmholtz Center Munich at the University Hospital Carl Gustav Carus and Faculty of Medicine of the TU Dresden, Dresden, Germany ${ }^{10}$ German Center for Diabetes Research (DZD e.V.), Neuherberg, Germany

tShared last authors

${ }^{*}$ Corresponding authors

Fluorescence microscopy is a key driver of discoveries in the life-sciences, with observable phenomena being limited by the optics of the microscope, the chemistry of the fluorophores, and the maximum photon exposure tolerated by the sample. These limits necessitate trade- offs between imaging speed, spatial resolution, light exposure, and imaging depth. In this work we show how image restoration based on deep learning extends the range of biological phenomena observable by microscopy. On seven concrete examples we demonstrate how microscopy images can be restored even if 60 -fold fewer photons are used during acquisition, how near isotropic resolution can be achieved with up to 10 -fold under-sampling along the axial direction, and how tubular and granular structures smaller than the diffraction limit can be resolved at 20-times higher frame-rates compared to state-of-the-art methods. All developed image restoration methods are freely available as open source software in Python, FIJI, and KNIME.

\section{Introduction}

Fluorescence microscopy is an indispensable tool in the life sciences for investigating the spatio-temporal dynamics of cells, tissues, and developing organisms. Recent advances, such as light-sheet microscopy [1-3], structured illumination microscopy [4, 5], and super-resolution microscopy [6-8] enable time resolved volumetric imaging of biological processes within cells at high resolution. The quality at which these processes can be faithfully recorded, however, is not only determined by the spatial resolution of the used optical device, but also by the desired temporal resolution, the total duration of 
an experiment, the required imaging depth, the achievable fluorophore density, bleaching, and photo-toxicity $[9,10]$. These aspects cannot all be optimized at the same time - one must make trade-offs, for example, sacrificing signal-to-noise ratio by reducing exposure time in order to gain imaging speed. Such trade-offs are often depicted by a design-space that has resolution, speed, light-exposure, and imaging depth as its dimensions (Figure 1a) with the volume being limited by the maximal photon budget compatible with sample health $[11,12]$.

These trade-offs can be addressed by optimizing the microscopy hardware, yet there are physical limits that cannot easily be overcome. Therefore, computational procedures to improve the quality of acquired microscopy images are becoming increasingly important. Super resolution microscopy [4, 13-16], deconvolution [17-19], surface projection algorithms [20,21], and denoising methods [22-24] are examples of sophisticated image restoration algorithms that can push the limit of the design-space, and thus allow one to recover important biological information that would be inaccessible by imaging alone. Most common image restoration problems, however, have multiple possible solutions, and require additional assumptions in order to select one solution as the final restoration. These assumptions are typically general, e.g. requiring certain level of smoothness of the restored image, and therefore are not dependent on the specific content of the images to be restored. Intuitively, a method that leverages available knowledge about the data at hand ought to reach superior restoration results.

Deep Learning (DL) is such a method, since it can learn to perform complex tasks on specific data $[25,26]$. In biology, DL methods have for instance been applied to the automatic extraction of connectomes from large electron microscopy data [27], for classification of image-based high- content screens [28], fluorescence signal prediction from label-free images $[29,30]$, resolution enhancement in histopathology [31], or for single molecule localization in super resolution microscopy $[32,33]$. However, the direct application of DL methods to image restoration tasks in fluorescence microscopy is complicated by the absence of training data sets and the fact that it is impossible to generate them manually.

In this paper, we present a solution to the problem of missing training data for DL in fluorescence microscopy by developing strategies to generate such data. This enables us to apply neural networks to image restoration tasks, such as image denoising, surface projection, recovery of isotropic resolution, and the restoration of sub-diffraction structures. We show, in a variety of imaging scenarios, that trained content-aware restoration (CARE) networks produce results that were previously unobtainable. This means that the application of CARE to biological images allows to transcend the limitations of the design-space (Figure 1a), pushing the limits of the possible in fluorescence microscopy through machine learned image computation.

\section{Results}

In fluorescence microscopy one is often forced to image samples at low signal intensities, resulting in low signal-to-noise ratio (SNR) images that are difficult to analyze. One way to improve SNR is to increase laser power or exposure times which is usually detrimental to the sample, limiting the possible duration of the recording and 
introducing artifacts due to photo-damage. An alternative solution is to image at low SNR, and later computationally restore acquired images. Classical approaches, such as non-local-means denoising [22], can in principle achieve this, but without leveraging available knowledge about the data at hand.

Image Restoration with Physically Acquired Training Data. To demonstrate the power of machine learning in biology, we developed content-aware image restoration (CARE). We first demonstrate the utility of CARE on microscopy acquisitions of the flatworm Schmidtea mediterranea, a model organism for studying tissue regeneration. This organism is exceptionally sensitive to even moderate amounts of laser light [34], suffering muscle flinching at desirable illumination levels even when anesthetized (Supp. Video 1). Using a laser power that reduces flinching to an acceptable level results in images with such low SNR that they are impossible to interpret directly. Consequently, live imaging of $S$. mediterranea has thus far been intractable.

To address this problem with CARE, we imaged fixed worm samples at several laser intensities. We acquired well-registered pairs of images, a low-SNR image at laser power compatible with live imaging, and a high-SNR image, serving as ground-truth $(c f$. Figure $1 \mathrm{~b}$ ). We then trained a convolutional neural network ${ }^{1}$ and applied the trained network to previously unseen live imaging data of $S$. mediterranea (Supp. Notes $1 \& 2$ ). We consistently obtained high quality restorations, even if the SNR of the images was very low, e.g. being acquired with a 60 -fold reduced light-dosage (Figure 1c \& d, Supp. Video 2, Supp. Figure 2-4). To quantify this observation, we measured the restoration error between prediction and ground-truth images for three different exposure and laser-power conditions. Both, the NRMSE ${ }^{2}$ and the SSIM ${ }^{3}$ measures of error improved considerably when compared to results obtained by several potent classical denoising methods (Figure 1d, Supp. Figure $3 \& 5$, Supp. Table 1). We further observed that already a small number of training images (e.g. 200 patches of size $64 \times 64 \times 16$ ) leads to an acceptable image restoration quality (Supp. Figure 6). Moreover, while training a CARE network can take several hours, the restoration time for a volume of size $1024 \times$ $1024 \times 100$ was less than 20 seconds on a single graphics processing unit ${ }^{4}$ In this case, CARE networks are able to take input data that are unusable for biological investigations and turn them into high-quality time-lapse data, providing the first practical framework for live-cell imaging of $S$. mediterranea.

We next asked whether CARE improves common downstream analysis tasks in live-cell imaging, such as nuclei segmentation. We used confocal microscopy recordings of developing Tribolium castaneum (red flour beetle) embryos, and as before trained a network on image pairs of samples acquired at high and low laser powers (Figure 1e). The resulting CARE network performs well even on extremely noisy, previously unseen live-imaging data (Supp. Notes 4, Supp. Video 3, Supp. Figure 7). In order to test the benefits of CARE for segmentation, we applied a simple nuclei segmentation pipeline to

\footnotetext{
${ }^{1}$ We use networks of moderate size $\left(\approx 10^{6}\right.$ parameters $)$ based on the U-net architecture $[35,36]$, together with a per-pixel similarity loss, e.g. absolute error (cf. Supp. Figure 1, Supp. Notes 2 and Supp. Table 3).

${ }^{2}$ Normalized root-mean-square error.

${ }^{3}$ Structural similarity index, measuring the perceived similarity between two images [37].

${ }^{4}$ We used a common consumer GPU (Nvidia GeForce GTX 1080 or Titan X) for all presented experiments.
} 
raw and restored image stacks of T. castaneum. The results show that, compared to manual expert segmentation, the segmentation accuracy (as measured with the standard SEG score [38]) improved from SEG $=0.47$ on the classically denoised raw stacks to $\mathrm{SEG}=0.65$ on the CARE restored volumes (Supp. Figure 8). Since this segmentation performance is achieved at significantly reduced laser power, the gained photon budget can now be spent on the imaging speed and light-exposure dimensions of the design-space. This means that Tribolium embryos, when restored with CARE, can be imaged longer and at higher frame rates, enabling improved tracking of cell lineages.

Encouraged by the performance of CARE on two independent denoising tasks, we asked whether such networks can also solve more complex, composite tasks. In biology it is often useful to image a 3D volume and project it to a 2D surface for analysis, for example when studying cell behavior in developing epithelia of the fruit fly Drosophila melanogaster $[39,40]$. Also in this context, it is beneficial to optimize the trade-off between laser-power and imaging speed, usually resulting in rather low-SNR images. Thus, this restoration problem is composed of projection and denoising, presenting the opportunity to test if CARE networks can deal with such composite tasks. For training, we again acquired pairs of low and high SNR 3D image stacks, and further generated 2D projection images from the high SNR stacks [20] that serve as ground-truth (Figure 2a). We developed a task-specific network architecture that consists of two jointly trained parts: a network for surface projection, followed by a network for image denoising (Figure 2b, Supp. Figure 9 and Supp. Notes 2). The results show that with CARE, reducing light dosage up to 10 -fold has virtually no adverse effect on the quality of segmentation and tracking results obtained on the projected 2D images with an established analysis pipeline [41] (Figure 2c \& d, Supp. Video 4, and Supp. Figure 10, 11 \& 12). Even for this complex task, the gained photon-budget can be used to move beyond the design-space, for example by increasing temporal resolution, and consequently improving the precision of tracking of cell behaviors during wing morphogenesis [41].

Image Restoration with Semi-synthetic Training Data. A common problem in fluorescence microscopy is that the axial resolution of volumetric acquisitions is significantly lower than the lateral resolution ${ }^{5}$. This anisotropy compromises the ability to accurately measure properties such as the shapes or volumes of cells. Anisotropy is caused by the inherent axial elongation of the optical point spread function (PSF), and the often low axial sampling rate of volumetric acquisitions required for fast imaging. For the restoration of isotropic image resolution, adequate pairs of training data cannot directly be acquired at the microscope. Rather, we took well-resolved lateral slices as ground-truth, and computationally modified them (i.e. applied a realistic imaging model, Supp. Notes 2) to resemble anisotropic axial slices of the same image stack. In this way, we generated matching pairs of images showing the same content at axial and lateral resolutions. These semi-synthetically generated pairs are suitable to train a CARE network that then restores previously unseen axial slices to nearly isotropic resolution (Figure 3a, Supp. Figure 13, Supp. Notes 2, and [43, 44]). In order to restore entire

\footnotetext{
${ }^{5}$ Some advanced modalities allow for (close to) isotropic acquisitions, e.g. multi-view light-sheet microscopy $[19,42]$.
} 
anisotropic volumes, we applied the trained network to all lateral image slices, taken in two orthogonal directions, averaged to a single isotropic restoration (Supp. Notes 2).

We applied this strategy to increase axial resolution of acquired volumes of fruit fly embryos [45], zebrafish retina [46], and mouse liver, imaged with different fluorescence imaging techniques. The results show that CARE improved the axial resolution in all three cases considerably (Figure 3b-d, Supp. Video 5 \& 6, and Supp. Figure 14 \& 15). In order to quantify this, we performed Fourier-spectrum analysis of Drosophila volumes before and after restoration, and showed that the frequencies along the axial dimension are fully restored, while frequencies along the lateral dimensions remain unchanged (Figure $3 \mathrm{~b}$ and Supp. Figure 16). Since the purpose of the fruit fly data is to segment and track nuclei, we applied a common segmentation pipeline [47] to the raw and restored images, and observed that the fraction of incorrectly identified nuclei was lowered from $1.7 \%$ to $0.2 \%$ (Supp. Notes 2, Supp. Figure $17 \& 18$ ). Thus, restoring anisotropic volumetric embryo images to effectively isotropic stacks, leads to improved segmentation, and will enable more reliable extraction of developmental lineages.

While isotropic images facilitate segmentation and subsequent quantification of shapes and volumes of cells, vessels, or other biological objects of interest, higher imaging speed enables imaging of larger volumes and their tracking over time. Indeed, respective CARE networks deliver the desired axial resolution with up to 10 -fold fewer axial slices (Figure 3 c \& d, see Supp. Figure 19 for comparison with classical deconvolution), allowing one to reach comparable results ten times faster. We quantified the effect of subsampling on raw and restored volumes with respect to restorations of isotropically sampled volumes for the case of the liver data (Figure 3d, Supp. Figure 20). Finally, we observed that for two-channel data sets like the zebrafish, networks learned to exploit correlations between channels, leading to a better overall restoration quality compared to results based on individual channels (Supp. Figure 15).

Image Restoration with Synthetic Training Data. Having seen the potential of using semi- synthetic training data for CARE, we next investigated whether reasonable restorations can be achieved even from synthetic image data alone, i.e. without involving real microscopy data during training.

In most of the previous applications, one of the main benefits of CARE networks was improved imaging speed. Many biological applications additionally require resolving sub-diffraction structures in the context of live-cell imaging. Super-resolution imaging modalities achieve the necessary resolution, but suffer from low acquisition rates. On the other hand, widefield imaging offers the necessary speed, but lacks the required resolution. We therefore tested whether CARE can computationally resolve subdiffraction structures using only widefield images as input. Note that this is a fundamentally different approach compared to recently proposed methods for single molecule localization microscopy that reconstruct a single super-resolved image from multiple diffraction limited input frames using deep-learning [32, 33]. To this end, we developed synthetic generative models of tubular and point-like structures that are commonly studied in biology. In order to obtain synthetic image pairs for training, we used these generated structures as ground- truth, and computationally modified them to resemble actual microscopy data (Supp. Notes 2, Supp. Figure 21). Specifically, we created synthetic ground-truth images of tubular meshes resembling microtubules, and 
point-like structures of various sizes mimicking secretory granules. Then we computed synthetic input images by simulating the image degradation process by applying a PSF, camera noise, and background auto-fluorescence (Figure 4a, Supp. Notes 2, and Supp. Figure 21). Finally, we trained a CARE network on these generated image pairs, and applied it to 2-channel widefield time-lapse images of rat INS-1 cells where the secretory granules and the microtubules were labeled (Figure $4 \mathrm{~b}$ ). We observed that the restoration of both microtubules and secretory granules exhibit a dramatically improved resolution, revealing structures imperceptible in the widefield images (Supp. Video 7, and Supp. Figure 22). To substantiate this observation, we compared the CARE restoration to the results obtained by deconvolution, which is commonly used to enhance widefield images (Figure 4b). Line profiles through the data show the improved performance of the CARE network over deconvolution (Figure 4b). We additionally compared results obtained by CARE with those from super-resolution radial fluctuations (SRRF [14]), a state-of-the-art method for reconstructing super-resolution images from widefield time-lapse data. We applied both methods on time-lapse widefield images of GFP-tagged microtubules in HeLa cells. The results show that both CARE and SRRF are able to resolve qualitatively similar microtubular structures (Figures 4c, Supp. Video 8). However, CARE reconstructions enable to image at least 20 times faster, since they are computed from a single average of up to 10 consecutive raw images while SRRF required about 200 consecutive widefield frames. We also used SQUIRREL [48] to quantify the error for both methods and observed that CARE generally produced better results, especially in image regions containing overlapping structures of interest (Figure 4d, Supp. Figure 23).

Taken together, these results suggest that CARE networks can enhance widefield images to a resolution usually only obtainable with super-resolution microscopy, yet at considerably higher frame rates.

239 Reliability of Image Restoration. We have shown that CARE networks perform well on 240 a wide range of image restoration tasks, opening new avenues for biological

241 observations (Supp. Table 2). However, as for any image processing method, the issue of 242 reliability of results needs to be addressed.

243 CARE networks are trained for a specific biological organism, fluorescent marker, and 244 microscope setting. When applying a network to data it was not trained for, results are 245 likely to suffer in quality ${ }^{6}$. Nevertheless, we observed only minimal "hallucination" 246 effects, where structures seen in the training data erroneously appear in restored 247 images (see Supp. Figure 24 \& 25). In Supp. Figure 25a we show the two strongest 248 errors across the entire body of available image data.

249 Nevertheless it is essential to identify cases where the above-mentioned problems 250 occur. To enable this, we changed the last network layer so that it predicts a probability 251 distribution ${ }^{7}$ for each pixel (Figure 5a, Methods, and Supp. Notes 3. For probabilistic

\footnotetext{
${ }^{6}$ As is the case for any (supervised) method based on machine learning.

${ }^{7}$ We chose a Laplace distribution for simplicity and robustness, see Supp. Notes 3.
} 
CARE networks, the mean of the distribution is used as the restored pixel value, while the width (variance) of each pixel distribution encodes the uncertainty of pixel predictions. Intuitively, narrow distributions signify high confidence, whereas broad distributions indicate low confidence pixel predictions. This allows us to provide per-pixel confidence intervals of the restored image (Figure 5a, and Supp. Figure $26 \& 27$ ). We observed that variances tend to increase with restored pixel intensities. This makes it hard to intuitively understand which areas of an restored image are reliable or unreliable from a static image of per-pixel variances. Therefore, we visualize the uncertainty in short video sequences, where pixel intensities are randomly sampled from their respective distributions (Supp. Video 9). We additionally reasoned that by analyzing the consistency of predictions from several trained models we can assess their reliability. To that end, we train ensembles (Figure $5 \mathrm{~b}$ ) of about 5 CARE networks on randomized sequences of the same training data. We introduced a measure $D$ that quantifies the probabilistic ensemble disagreement per pixel (Methods, Supp. Notes 3). D takes values between 0 and 1, with higher values signifying larger disagreement, i.e. smaller overlap among the distributions predicted by the networks in the ensemble. Using fly wing denoising as an example, we observed that in areas where different networks in an ensemble predicted very similar structures, the disagreement measure D was low (Figure 5c, top row), whereas in areas where the same networks predicted obviously dissimilar solutions, the corresponding values of D were large (Figure 5c, bottom row). Therefore, training ensembles of CARE networks is useful to detect problematic image areas that cannot reliably be restored ${ }^{8}$.

Availability of CARE. Code for network training and prediction (in Python/TensorFlow) is publicly available 9 . Furthermore, to make our restoration models readily available, we developed user- friendly FIJI plugins and KNIME workflows (Supp. Figure 29 \& 30).

\section{Discussion}

We have introduced content-aware image restoration (CARE) networks designed to restore fluorescence microscopy data. A key feature of our approach is that generating training data does not require laborious manual training data generation. With CARE, flatworms can be imaged without unwanted muscle contractions, beetle embryos can be imaged much gentler and therefore longer and faster, large tiled scans of entire Drosophila wings can be imaged and simultaneously projected at dramatically increased temporal resolution, isotropic restorations of embryos and large organs can be computed from existing anisotropic data, and sub-diffraction structures can be restored from widefield systems at high frame rate. In all these examples, CARE allows to invest the photon budget saved during imaging into improvement of acquisition parameters relevant for a given biological problem, such as speed of imaging, photo-toxicity, isotropy, or resolution.

Whether an experimentalist is willing to make the above mentioned investment, depends on her trust that a CARE network is accurately restoring the image. This is a

\footnotetext{
${ }^{8}$ Another example for the utility of ensemble disagreement can be found in Supp. Figure 28.

${ }^{9}$ https://github.com/CSBDeep/CSBDeep
} 
valid concern, that applies to every image restoration approach. What sets CARE apart is the availability of additional readouts, i.e. per-pixel confidence intervals and ensemble disagreement scores, that allow users to identify image regions where restorations might not be accurate.

297

We have shown multiple examples where image restoration with CARE networks

298 positively impacts downstream image analysis, such as segmentation and tracking of cells needed for extracting developmental lineages. Interestingly, in the case of Tribolium, CARE improved segmentation by efficient denoising, whereas in the case of Drosophila, the segmentation was improved by increasing the isotropy of volumetric acquisitions. These two benefits are not mutually exclusive and could very well be combined. In fact, we have shown on data from developing Drosophila wings, that composite tasks can jointly be trained. Future explorations of jointly training composite networks will further broaden the applicability of CARE to complex biological imaging problems (cf. [49]).

Yet, CARE networks cannot be applied to all existing image restoration problems. For instance, the proposed isotropic restoration relies on the implicit assumption that structures of interest do appear in arbitrary orientations and that the PSF is constant throughout the image volume ${ }^{10}$. Additionally, due to the non-linear nature of neural network predictions, CARE must not be used for intensity- based quantifications, e.g. fluorophore counting. Furthermore, the disagreement score we introduced may be useful to additionally identify instances where training and test data are incompatible, i.e. when a CARE network is applied on data that contains biological structures absent from the training set.

316 Overall, our results show that fluorescence microscopes can, in combination with 317 content- aware restorations, operate at higher frame-rates, shorter exposures, and 318 lower light intensities, while reaching higher resolution, and thereby improving 319 downstream analysis. The technology described here is readily accessible to the scientific community through the open source tools we provide. We predict that the current explosion of image data diversity and the ability of CARE networks to automatically adapt to various image contents, will make such learning approaches prevalent for biological image restoration and will open new windows into the inner workings of biological systems across scales.

\section{Acknowledgements}

The authors want to thank Philipp Keller (Janelia) who provided Drosophila data. We thank Suzanne Eaton (MPI- CBG), Franz Gruber and Romina Piscitello for sharing the expertise in fly imaging and providing fly lines. We thank Anke So"nmez for cell culture work. We thank Marija Matejcic (MPI-CBG) for generating and sharing the LAP2b transgenic line Tg(bactin:eGFP-LAP2b). We thank Benoit Lombardot from the Scientific Computing Facility (MPI-CBG) for technical support. We thank the following Services and Facilities of the MPI-CBG for their support: Computer Department, Light Microscopy Facility and Fish Facility. This work was supported by the German Federal Ministry of Research and Education (BMBF)

\footnotetext{
10 This assumption is only approximately true, becoming increasingly worse when imaging deeper into tissues.
} 
under the codes 031L0102 (de.NBI) and 031L0044 (Sysbio II) and the Deutsche Forschungsgemeinschaft (DFG) under the code JU 3110/1-1. M.S. was supported by the German Center for Diabetes Research (DZD e.V.). TB was supported by an ELBE postdoctoral fellowship and an Add-on Fellowship for Interdisciplinary Life Sciences awarded by the Joachim Herz Stiftung. R.H. and S.C. were supported by the following grants: UK BBSRC (BB/M022374/1; BB/P027431/1; BB/R000697/1), UK MRC (MR/K015826/1) and Wellcome Trust (203276/Z/16/Z).

\section{Author Contributions}

M.W. and L.R. initiated the research. M.W. and U.S. designed and implemented the training and validation methods. U.S., M.W., and F.J. designed and implemented the uncertainty readouts. T.B., A.M., A.D., S.C., F.S.M., R.H., M.R.M., and A.J. collected experimental data. A.D., C.B., and F.J. performed cell segmentation analysis. T.B. performed analysis on flatworm data. U.S. and M.W. designed and developed the Python package. F.J., B.W., and D.S. designed and developed the FIJI and KNIME integration. E.W.M supervised the project. F.J., M.W., P.T., L.R., U.S., \& E.W.M wrote the manuscript, with input from all authors.

\section{Competing interests}

The authors declare no competing interests.

\section{Data availability}

Training and test data for all experiments presented can be found at https://publications.mpi-cbg.de/ publications-sites/7207.

\section{References}

[1] J. Huisken et al. "Optical sectioning deep inside live embryos by selective plane illumination microscopy.” In: Science 305 (5686 2004),

[2] R. Tomer et al. "Quantitative high-speed imaging of entire developing embryos with simultaneous multiview light-sheet microscopy.” In: Nature Methods 9.7 (July 2012),

[3] B.-C. Chen et al. "Lattice light-sheet microscopy: imaging molecules to embryos at high spatiotemporal resolution.” In: Science 346.6208 (Oct. 2014),

[4] M. G. Gustafsson. "Surpassing the lateral resolution limit by a factor of two using structured illumination microscopy". In: Journal of Microscopy 198.2 (2000),

[5] R. Heintzmann and M. G. Gustafsson. "Subdiffraction resolution in continuous samples”. In: Nature Photonics 3.7 (2009),

[6] E. Betzig et al. "Imaging intracellular fluorescent proteins at nanometer resolution". In: Science 313.5793 (2006),

[7] M. J. Rust, M. Bates, and X. Zhuang. "Sub-diffraction-limit imaging by stochastic optical reconstruction microscopy (STORM)”. In: Nature Methods 3.10 (2006),

[8] K. I. Mortensen et al. "Optimized localization analysis for single-molecule tracking and super-resolution microscopy”. In: Nature Methods 7.5 (Apr. 2010),

[9] J. Icha et al. "Phototoxicity in live fluorescence microscopy, and how to avoid it". In: Bioessays 39.8 (2017). 
370 [10] P. P. Laissue et al. "Assessing phototoxicity in live fluorescence imaging”. In: Nature

371 Methods 14.7 (2017),

372 [11] J. B. Pawley. "Fundamental limits in confocal microscopy". In: Handbook of

373 Biological Confocal Microscopy. Springer, 2006,

374 [12] N. Scherf and J. Huisken. "The smart and gentle microscope". In: Nature

375 biotechnology 33.8 (2015),

376 [13] M.Mu“lleretal.“Open-sourceimagereconstructionofsuper-

377 resolutionstructuredillumination microscopy data in ImageJ". In: Nature

378 Communications 7 (2016).

379 [14] N. Gustafsson et al. "Fast live-cell conventional fluorophore nanoscopy with ImageJ

380 through super-resolution radial fluctuations". In: Nature Communications 7 (2016).

381 [15] T. Dertinger et al. "Superresolution optical fluctuation imaging (SOFI)". In: Nano-

382 Biotechnology for Biomedical and Diagnostic Research. Springer, 2012,

383 [16] K. Agarwal and R. Macha'n. "Multiple signal classification algorithm for super-

384 resolution fluorescence microscopy". In: Nature Communications 7 (2016),

385 [17] W. H. Richardson. "Bayesian-based iterative method of image restoration". In:

386 Journal of the Optical Society of America 62.1 (1972),

387 [18] M. Arigovindan et al. "High-resolution restoration of 3D structures from widefield 388 images with extreme low signal-to-noise-ratio". In: Proceedings of the National Academy 389 of Sciences 110.43 (2013),

390 [19] S. Preibisch et al. "Efficient Bayesian-based multiview deconvolution." In: Nature 391 Methods 11 (6 2014),

392 [20] C. Blasse et al. "PreMosa: extracting 2D surfaces from 3D microscopy mosaics". In: 393 Bioinformatics (2017),

394 [21] A. Shihavuddin et al. "Smooth 2D manifold extraction from 3D image stack". In:

395 Nature Communications 8 (2017).

396 [22] A. Buades, B. Coll, and J.-M. Morel. "A non-local algorithm for image denoising". In:

397 IEEE Conference on Computer Vision and Pattern Recognition. Vol. 2. 2005,

398 [23] K. Dabov et al. "BM3D image denoising with shape-adaptive principal component 399 analysis". In: SPARS'09-Signal Processing with Adaptive Sparse Structured

400 Representations. 2009.

401 [24] H. Morales-Navarrete et al. "A versatile pipeline for the multi-scale digital

402 reconstruction and quantitative analysis of 3D tissue architecture". In: Elife 4 (2015), 
403 [25] Y. LeCun et al. "Gradient-based learning applied to document recognition". In:

404 Proceedings of the IEEE 86.11 (1998),

405 [26] Y. LeCun, Y. Bengio, and G. Hinton. “Deep learning”. In: Nature 521.7553 (2015),

406 [27] T. Beier et al. "Multicut brings automated neurite segmentation closer to human 407 performance". In: Nature Methods 14.2 (2017),

408 [28] J. C. Caicedo et al. "Data-analysis strategies for image-based cell profiling". In:

409 Nature Methods 14.9 (2017),

410 [29] C. Ounkomol et al. "Label-free prediction of three-dimensional fluorescence images

411 from transmitted-light microscopy”. In: Nature Methods (2018).

412 [30] E. M. Christiansen et al. "In silico labeling: Predicting fluorescent labels in 413 unlabeled images”. In: Cell 173.3 (2018),

414 [31] Y. Rivenson et al. "Deep learning microscopy”. In: Optica 4.11 (2017),

415 [32] E. Nehme et al. "Deep-STORM: super-resolution single-molecule microscopy by 416 deep learning". In: Optica 5.4 (2018),

417 [33] W. Ouyang et al. "Deep learning massively accelerates super-resolution 418 localization microscopy”. In: Nature biotechnology (2018).

419 [34] N. Shettigar et al. "Hierarchies in light sensing and dynamic interactions between 420 ocular and extraocular sensory networks in a flatworm". In: Science Advances 3.7

421 (2017),

422 [35] O. Ronneberger, P. Fischer, and T. Brox. "U-net: Convolutional networks for 423 biomedical image segmentation". In: International Conference on Medical Image

424 Computing and Computer-Assisted Intervention. 2015.

425 [36] X. Mao, C. Shen, and Y.-B. Yang. "Image Restoration Using Very Deep Convolutional 426 Encoder-Decoder Networks with Symmetric Skip Connections". In: Advances in Neural 427 Information Processing Systems 29. 2016,

428 [37] Z. Wang et al. "Image quality assessment: from error visibility to structural 429 similarity”. In: IEEE Transactions on Image Processing 13.4 (2004),

430 [38] V. Ulman et al. "An objective comparison of cell-tracking algorithms". In: Nature 431 Methods (2017).

432 [39] B. Aigouy et al. "Cell flow reorients the axis of planar polarity in the wing 433 epithelium of Drosophila”. In: Cell 142.5 (2010),

434 [40] R. Etournay et al. "Interplay of cell dynamics and epithelial tension during 435 morphogenesis of the Drosophila pupal wing”. In: Elife 4 (2015), 
436 [41] R. Etournay et al. "TissueMiner: A multiscale analysis toolkit to quantify how

437 cellular processes create tissue dynamics". In: Elife 5 (2016),

438 [42] R. K. Chhetri et al. "Whole-animal functional and developmental imaging with

439 isotropic spatial resolution.” In: Nature Methods 12.12 (Dec. 2015),

440 [43] M. Weigert et al. "Isotropic Reconstruction of 3D Fluorescence Microscopy Images

441 Using Convolutional Neural Networks". In: International Conference on Medical Image

442 Computing and Computer-Assisted Intervention. 2017.

443 [44] L. Heinrich, J. A. Bogovic, and S. Saalfeld. "Deep Learning for Isotropic Super-

444 Resolution from Non-isotropic 3D Electron Microscopy". In: International Conference on

445 Medical Image Computing and Computer-Assisted Intervention. 2017.

446 [45] L. A. Royer et al. "Adaptive light-sheet microscopy for long-term, high-resolution

447 imaging in living organisms”. In: Nature biotechnology 34.12 (2016),

448 [46] J. Icha et al. "Independent modes of ganglion cell translocation ensure correct

449 lamination of the zebrafish retina”. In: Journal of Cell Biology 215.2 (2016),

450 [47] C. Sommer et al. "Ilastik: Interactive learning and segmentation toolkit". In: IEEE

451 International Symposium on Biomedical Imaging: From Nano to Macro. 2011.

452 [48] S. Culley et al. "Quantitative mapping and minimization of super-resolution optical 453 imaging artifacts". In: Nature methods 15.4 (2018),

454 [49] L. Sui et al. "Differential lateral and basal tension drives epithelial folding through 455 two distinct mechanisms". In: Nature Communications (in press) (2018), 

and light exposure need to be found due to the constraints of the maximal photon budget a sample permits. Image restoration enlarges this design-space. (b) Overview of the proposed pipeline for image denoising. Pairs $\left(\mathrm{x}_{\mathrm{i}}, \mathrm{y}_{\mathrm{i}}\right)$ of registered high signal-to-noise (SNR) and low SNR volumes are acquired at the microscope. A convolutional neural network is trained to restore $y_{\mathrm{i}}$ from $\mathrm{x}_{\mathrm{i}}$. The trained CARE network can then be applied to previously unseen low SNR images $\tilde{x^{2}}$ yielding y. (c) Input data and restorations for nucleus-stained (RedDot1) flatworm (Schmidtea mediterranea). Shown are a single image-plane of a raw input stack (top row), the output of non-local-means denoising (NLM [22], second row), the network prediction (third row), and the high SNR gold-standard/ground-truth (bottom row). Input images were acquired with 60 -fold reduced light-dosage compared to ground-truth images. A comparison to more denoising methods can be found in Supp. Figure 5. (d) Prediction error for data from (c) at 3 imaging conditions $\mathrm{C} 1-\mathrm{C} 3$. Box- dot plots ( $\mathrm{n}=20$ per condition) show normalized root-mean-squared error (NRMSE) and structural similarity (SSIM, higher is better) for the input, for the denoising baseline (NLM), and for network restorations. Boxes show interquartile range (IQR), line signify median, and whiskers extend to 1.5 IQR. (e) Input data and restorations for a nucleus-labeled (EFA::nGFP) red flour beetle (Tribolium castaneum) embryo. Figure structure as in (c).

Figure 2: Joint surface projection and denoising. (a) Schematic of the composite task at hand. A single cell layer of interest of a Drosophila wing is embedded in an imaged 3D volume. Live-cell imaging imposes low-light acquisitions to avoid photo-toxicity. The desired pipeline extracts and denoises the 2D tissue layer from low SNR input volumes. (b) The proposed CARE network first projects the data and then performs a 2D denoising step. (c) Restoration results on E-cadherin labeled fly wing data acquired with a spinning disk microscope. Shown are a max-projection of the raw input data (top row), a surface projection baseline obtained by a state-of-the-art method PreMosa [20] (second row), CARE network results (third row), and ground-truth projections obtained by applying PreMosa on a very high laserpower acquisition of the same sample (bottom row). (d) Prediction error for 3 imaging conditions (C1C3, see Methods). Box-dot plots ( $\mathrm{n}=26$ per conditions) show normalized root-mean-squared error (NRMSE) and structural similarity (SSIM, higher is better) for results obtained using PreMosa (blue), PreMosa with additional denoising (NLM [22], green), and CARE network results (orange). Boxes show interquartile range (IQR), line signify median, and whiskers extend to 1.5 IQR. Comparison to additional baselines can be found in Supp. Figure 11.

Figure 3: Isotropic restoration of 3D volumes. (a) Schematic of the semi-synthetic generation of training data. Lateral slices of the raw data (green) are used as ground-truth, which are then synthetically down-sampled and convolved with the rotated PSF of the used microscope. This results in corresponding anisotropic slices (black) with similar resolution as the raw axial slices (orange). (b) Application to timelapse acquisitions of Drosophila melanogaster [45]. Shown are three areas of the raw axial input data (top row), and their respective isotropic restorations (bottom row). Additionally the Fourier-spectrum of raw and restored images illustrate how missing spectral components are recovered. (c) An axial optical slice through a developing zebrafish (Danio rerio) eye. Shown are the anisotropic raw data (top row) and isotropic restoration (bottom row). Nuclei are stained with DRAQ5 (magenta) and the nuclear envelope is labeled with GFP-LAP2b (green). A comparison to deconvolution can be found in Supp. Figure 11. (d) An axial optical slice through mouse liver tissue. Shown are the anisotropic raw data (with a subsampling of $\sigma=8$, top row) and the isotropic restoration by the network (middle row). Nuclei and membranes of hepatocytes are labeled with DAPI and Phalloidin, respectively, and imaged in a single channel. Plots show the effect of increasing levels of axial subsampling of raw (blue) and isotropically restored (orange) volumes. We plot the normalized root-mean-squared error (NRMSE) and structural similarity (SSIM, higher is better) with respect to our restorations of the shown isotropically sampled raw data. Details on data and training can be found in Supp. Table 3).

Figure 4: Resolving sub-diffraction structures at high frame rates. (a) Schematic of the fully-synthetic training pipeline. Synthetic images of tubular and point-like structures (not shown) were computationally generated as ground- truth data, and have further been processed to resemble microscopy data. CARE networks were trained on such data and later applied on real microscopy images of diffraction-limited tubular and dot-like structures. (b) Raw widefield images of rat secretory granules (pEG-hIns-SNAP, magenta) and microtubules (SiR-tubulin, green) in insulin-secreting INS-1 cells (top row), the corresponding network restorations (second row), and a deconvolution result of the raw image as a baseline (bottom row). Line-plots show image intensities along the dashed lines in the top panels. (c) 
514 GFP-tagged microtubules in HeLa cells. Raw input image (top row), network restorations (second row), 515 super-resolution images created by the state-of-the-art method SRRF [14] (bottom row). Line-plots show 516 image intensities along the dashed lines in the top panels. (d) Error quantification via SQUIRREL [48] for 517 network results and the results obtained by SRRF. Shown are error maps corresponding to the dashed box 518 in (c) and the resolution scaled error (RSE) for 20 consecutive frames. The data shown in (c) corresponds 519 to frame 1.

520 Figure 5: Reliability readouts for content-aware image restorations. (a) For every pixel of a restored 521 image, CARE networks can predict a (Laplace) distribution parameterized by its mean $\mu$ and scale $\sigma$ (top). 522 These distributions provide pixel-wise confidence intervals (bottom), here shown for a surface projection and denoising network ( $c f$. Figure 2). The line-plot shows the predicted mean (blue), the $90 \%$ confidence interval (light-blue), and corresponding ground-truth intensities (dashed red) along the yellow dashed line in the image on the left. (b) Multiple independently-trained cARE networks are combined to form an ensemble, resulting in an ensemble distribution and an ensemble disagreement measure $D \in[0,1]$. (c) Ensemble predictions can vary, especially on challenging image regions. Shown are two examples for a surface projection and denoising ensemble of 4 networks (rows). From left to right we show: maximum projection of input data, predictions of the four networks of the ensemble, the pixel-wise ensemble mean, and the ensemble disagreement measure. While the top row shows an image region with low ensemble disagreement, the bottom row shows a region where individual network predictions differ, resulting in a high disagreement score in respective image areas. 
535 For each of the described experiments and restoration modalities, we (i) imaged or 536 generated suitable training data, (ii) trained a neural network (or ensemble of 537 networks), and (iii) applied the trained network and quantified/reported the results.

538 Network architecture and training: For all experiments (except fly wing projection) 539 we used residual versions of the U-Net architecture [50] in 3D or 2D (Supp. Figure 1, 540 Supp. Figure 13). For the fly wing projection task, we used a two stage architecture 541 combining a projection and a denoising sub-network (Supp. Figure 9). All restoration 542 experiments were performed in Python using Keras [51] and TensorFlow [52]. Source 543 code for training and prediction, example applications, and documentation can be found 544 at http://csbdeep.bioimagecomputing.com/doc/. The training details for each 545 restoration experiment (e.g. number of used images, network hyper- parameters) are 546 listed in Supp. Table 3 and are described in Supp. Notes 2.

547 Image normalization: For training, prediction, and evaluation it is important to 548 normalize the input images to a common intensity range. We used percentile-based 549 normalization, typically using percentile ranks $\mathrm{p}_{\text {low }} \in(1,3)$ for determining the lowest 550 value and $p_{\text {high }} \in(99.5,99.9)$ for the highest value. All image pixels are then affinely 551 scaled, such to the lowest and highest values are converted to 0 and 1, respectively. For 552 a given image $y$, the percentile-normalized image will be denoted by $N\left(y, p_{\text {low }}, p_{\text {high }}\right)$.

553 Quantification of restoration errors: Since the image $y$ predicted by any restoration 554 method (CARE or any compared baseline) and the corresponding ground-truth image $y_{0}$ 555 typically differ in the dynamic range of their pixel values, they have to be normalized to 556 a common range first. To that end, we first percentile-normalize the ground-truth image $557 y_{0}$ as described before with $\mathrm{p}_{\text {low }}=0.1$ and $\mathrm{p}_{\text {high }}=99.9$. Secondly, we use a transformation $558 \varphi(y)=\alpha y+\beta$ that affinely scales and translates every pixel of the restored image 559 based on parameters

$560 \alpha, \beta=\operatorname{argmin} \operatorname{MSE}\left(N\left(y_{0}, 0.1,99.9\right), \alpha^{\prime} y+\beta^{\prime}\right)$ with $\operatorname{MSE}(u, v)=\frac{1}{N} \sum_{i=1}^{N}\left(u_{i}-v_{i}\right)^{2}$ 561 That is, $\alpha, \beta$ are chosen such that the mean squared error (MSE) between $\varphi(y)$ and $N\left(y_{0}\right.$, $5620.1,99.9$ ) is minimal (note that $\alpha, \beta$ can be easily computed in closed-form). All final 563 error metrics, such as normalized root-mean-squared error (NRMSE) or structural 564 similarity (SSIM, [53]) were computed on images normalized in this way. More details 565 can be found in Supp. Notes 2.

566 Planaria denoising. Planaria (Schmidtea mediterranea) were cultured at $20^{\circ} \mathrm{C}$ in 567 planarian water[54] and fed with organic bovine liver paste. To label nuclei, $S$. 568 mediterranea samples were stained for 15 hours in planarian water supplemented with $5692 \times$ RedDot1 and 1\% (v/v) DMSO. For training data acquisition, planaria were 570 euthanized with $5 \% \mathrm{w} / \mathrm{v} \mathrm{N}$-Acetyl-L-cysteine in PBS and subsequently fixed in $4 \% \mathrm{w} / \mathrm{v}$ 571 paraformaldehyde in PBS. For time lapse recordings RedDot1 stained planaria were 572 anesthetized for $1 \mathrm{hr}$ with $0.019 \% \mathrm{w} / \mathrm{v}$ Linalool prior mounting which was maintained 573 throughout the course of the live imaging experiments. A 5 min incubation in $0.5 \% \mathrm{w} / \mathrm{v}$ 574 pH neutralized N-Acetyl-L-cysteine was used to remove the animal's mucus prior 575 mounting. For imaging, fixed or live animals were mounted in refractive index matched $576 \quad 1.5 \%$ agarose ( $50 \% \mathrm{w} / \mathrm{v}$ Iodixanol) to enhance signal quality at higher imaging depths 
577 as previously described in [54]. For imaging, a spinning disc confocal microscope with a $57830 \mathrm{x} / 1.05$ silicon oil immersion objective and $640 \mathrm{~nm}$ excitation wavelength was used.

579 We used 4 different laser-powers/exposure-times imaging conditions GT (ground-

580 truth) and C1-C3, specifically $2.31 \mathrm{~mW} / 30 \mathrm{~ms}$ (GT), $0.12 \mathrm{~mW} / 20 \mathrm{~ms}$ (C1), $0.12 \mathrm{~mW} / 10 \mathrm{~ms}$

581 (C2), and $0.05 \mathrm{~mW} / 10 \mathrm{~ms}$ (C3). To ensure that corresponding image stacks are well

582 aligned, we interleaved all 4 different imaging conditions as different channels during

583 acquisition. In total, we acquired 96 stacks of average size $1024 \times 1024 \times 400$. From this

584 data we sampled around 17000 randomly positioned sub-volume pairs of size

$58564 \times 64 \times 16$ voxels. We evaluated our results on 20 previously unseen volumes of

586 Schmidtea mediterranea imaged at various developmental stages. As competing

587 denoising methods we chose lowpass filter, median filter, bilateral filter [55], non-local-

588 means denoising (NLM) [56], Total Variation denoising [57], BM3D [58], and BM4D

589 [59]. Please see Supp. Table 1 and Supp. Notes 2.3.1 for more details.

590 Tribolium denoising and segmentation. A EFA::nGFP transgenic line of Tribolium

591 castaneum was used for imaging embryonic development with labeled nuclei [60]. The

592 beetles were reared and embryos were collected according to standard protocols [61].

593 Imaging was done on a Zeiss 710 multiphoton laser scanning microscope using a $25 \mathrm{x}$

594 multi immersion objective. Similar to the planaria data set, we used 4 different laser-

595 power imaging conditions GT and C1-C3, specifically $20 \mathrm{~mW}$ (GT), $0.1 \mathrm{~mW}$ (C1), $0.2 \mathrm{~mW}$

596 (C2), and $0.5 \mathrm{~mW}$ (C3). For each condition we acquired 26 training stacks (of size

$597 \approx 700 \times 700 \times 50$ ) using different samples at different developmental stages. From that, we

598 randomly sampled around 15000 patches of size $64 \times 64 \times 16$ and trained a 3D network

599 as before. For testing, we used 6 additional volumes per condition, again acquired at

600 different developmental stages. As denoising baseline we again used non-local-means

601 denoising (NLM) [56]. Nuclei segmentation was performed using a thresholding-based

602 segmentation workflow. To create the segmentation ground-truth, we used ilastik [62]

603 to train a pixel-wise random forest classifier to distinguish nuclei and background pixels

604 in the high-SNR (GT) image, whose output was curated using a combination of

605 segmentation tools from SciPy [63], the 3D volume rendering software spimagine ${ }^{11}$, and

606 manual, pixel-wise corrections. To create segmentations for restorations (NLM or CARE)

607 of the low-SNR images (C2), we thresholded their intensities and labeled connected

608 components of pixels above the threshold as individual nuclei. The segmentation

609 accuracy was computed as the SEG score [64], which corresponds to the average

610 overlap of segmented regions with matched ground-truth regions ( $0 \leq \mathrm{SEG} \leq 1)$. More

611 details can be found in Supp. Notes 2.3.2.

612 Flywing projection, segmentation, and tracking. Drosophila melanogaster expressing

613 the membrane marker Ecad::GFP were raised under $25^{\circ} \mathrm{C}$ at standard conditions. Pupae

614 were collected and prepared for imaging as described in [65]. The dorsal side of the

615 pupal wing was imaged with a Yokogawa CSU-X1 spinning disk microscope using a

616 Zeiss LCI Plan-Neofluar 63x/1.3 Imm Corr objective. We acquired image stacks at 4

617 different conditions GT and C1-C3, with camera- exposure/laser-power of $240 \mathrm{~ms} / 20 \%$

618 (GT), 120ms/2\% (C1), 120ms/3\% (C2), 120ms/5\% (C3), where we again interleaved

619 all conditions during imaging. For each condition, we acquired 180 different 3D stacks

${ }^{11}$ https://github.com/maweigert/spimagine 
620 (of size $\approx 700 \times 700 \times 50$ ). As prediction target we used the surface projected 2D ground-

621 truth signal obtained via PreMosa [66] computed on data acquired with GT settings. For

622 training we sampled around 17000 random 3D patches of size $64 \times 64 \times 50$ from the

623 acquired stacks. For the composite task of joint projection and denoising, we designed a

624 stacked network architecture consisting of a projection and a denoising sub-network

625 (cf. Supp. Figure 9). We evaluated the restoration quality on 26 previously unseen

626 volumes, and compared results obtained with CARE against maximum projection (MIP),

627 smooth 2D manifold extraction (SME) [67], minimum cost surface projection

628 (GraphCut) [68, 69], and PreMosa [66]. For all competing methods (except CARE), we

629 additionally applied NLM denoising [56] to the respective output (cf. Supp. Figure 11).

630 To evaluate segmentation and tracking results on restored stacks, we used a time-

631 lapsed acquisition of 26 time-points imaged with the GT and C2 settings. To create a

632 binary segmentation of membrane and background regions, we used the a random

633 forest classifier (Trainable Weka Segmentation [70] plugin in Fiji [71]) that was trained

634 on images with 30 manually labeled cells (membrane contour and corresponding non-

635 membrane region inside) for both imaging settings. The probability maps generated by

636 the classifier were processed with Tissue Analyzer [72], a tool for tracking and

637 segmentation of cells in 2D epithelia, yielding a joint segmentation and tracking of cells

638 over all frames. For each frame we computed the SEG score based on the raw and

639 restored images with respect to the ground-truth ( $c f$. Supp. Figure 12). For more details,

640 see Supp. Notes 2.3.3.

641 Drosophila isotropic restoration and segmentation. All input stacks were provided

642 by the authors of [73], where histone-labeled Drosophila melanogaster embryos were

643 imaged using a light-sheet microscope. Note that this data set was already processed,

644 but still exhibited an anisotropic PSF and a 5-fold axial sub-sampling that translated

645 into a combined 4-6 fold decrease in axial resolution. We used the training data

646 strategy as described in Supp. Notes 2.4 and [74], where the 2D lateral slices are used as

647 ground-truth and are synthetically subsampled by $\sigma=5$ and blurred with the

648 theoretical PSF of the light-sheet microscope. We used 15 volumes from equally spaced

649 time-points during development (between embryo cellularization and germband

650 retraction), resulting in around 10000 training patches of size $128 \times 128$. As network

651 architecture we used a 2D U-Net (Supp. Figure 13). To quantify the restoration quality,

652 we computed the spectral isotropy ratio $\Phi$ as the ratio of spectral energy of the signal in

653 the Fourier-domain along the axial and lateral dimension. To evaluate a nuclei

654 segmentation task we used a crop of a densely populated center region containing

655 approximately 470 nuclei from an unseen test volume and generated ground-truth

656 segmentation masks with ilastik [62] employing extensive manual curation. We

657 compared the segmentability of network-restored images with bicubically upsampled

658 images by training a random forest classifier on both images using the GT masks as

659 target and generated instance segmentation via connected components of the

660 thresholded probability maps. To evaluate the segmentation, we computed a bipartite

661 matching between proposed and ground-truth nuclei instances ( $\mathrm{IoU} \geq 0.5$ ) and used the

662 fraction of unmatched nuclei as a measure of segmentation error.

663 Zebrafish retinal tissue isotropic restoration. Zebrafish (Danio rerio) imaging

664 experiments were performed with a transgenic zebrafish line Tg(bactin:eGFP-LAP2b)

665 that labels the nuclear envelope. Embryos were raised in E3 medium at $28.5^{\circ} \mathrm{C}$ and

666 treated with $0.2 \mathrm{mM}$ 1-phenyl-2-thiourea at $8 \mathrm{hpf}$ onward to delay pigmentation. 
667 Embryos were fixed at $24 \mathrm{hpf}$ in 4\% paraformaldehyde, permeabilized with $0.25 \%$

668 trypsin, and incubated with a far-red DNA stain (DRAQ5) for 2 days at $4^{\circ} \mathrm{C}$. Imaging of

669 agarose mounted embryos was performed on a spinning disk confocal (Andor

670 Revolution WD) with a $60 \mathrm{x} / 1.3$ objective, using excitation wavelengths of $\lambda=638 \mathrm{~nm}$

671 (DRAQ5) and $\lambda=488 \mathrm{~nm}$ (eGFP-LAP2b). Stack were acquired with 2 - $\mu \mathrm{m}$ steps, resulting

672 in an axially sub-sampling factor of $\sigma=10.2$. For generating training data we acquired 5

673 multi-channel volumes from which we extracted around 25000 lateral patches of size

$674128 \times 128 \times 2$, applied the corresponding theoretical PSF and subsampling model, yet

675 always keeping the information of both image channels. Network training was done as

676 before. To compare the restoration quality with classical deconvolution, we ran

677 Huygens (Scientific Volume Imaging, http://svi.nl) on the bicubic upsampled raw stacks

678 once with the actual PSF and once with an $\sigma$-fold down- and upsampled PSF (to account

679 for the additional blur related to upsampling). We used the following parameters from

680 Huygens: method $=$ MLE, number iteration $=70$, SNR parameter $=15$, quality threshold

$681=0.05$.

682 Mouse liver isotropic restoration. Mice livers were fixed through transcardial

683 perfusion with $4 \%$ paraformaldehyde and post-fixed overnight at $4^{\circ} \mathrm{C}$ with the same

684 solution. Tissue slices were optically cleared by a modified version of SeeDB [75] and

685 stained with DAPI (nuclei) and phalloidin (membrane). The samples were imaged using

686 a Zeiss LSM 780 NLO multiphoton laser- scanning microscope with a 63x/1.3 glycerol

687 immersion objective (Zeiss) using $780 \mathrm{~nm} 2$-photon excitation and isotropic voxel-size

688 of $0.3 \mu \mathrm{m}$. We acquired 8 stacks of mouse liver each of size $752 \times 752 \times 300$. For the range

689 of subsampling factors $\sigma=2 \ldots 16$ we created respective axial anisotropic stacks by

690 retaining only every $\sigma$-th axial slice from the original volumes to be restored later. For

691 each $\sigma$, we extracted around 15000 patches of size $128 \times 128$ from the given body of data

692 and trained a network as described before. We refer to Supp. Notes 2.4.3 for more

693 details.

694 INS-1celltubular/granules restoration. Rat insulin-secreting beta-cells(INS-1cells) were 695 cultured and transiently transfected with pEG-hIns-SNAP as previously described [76].

696 The cells were labeled with $1 \mu \mathrm{M}$ SNAP-Cell 505-Star (secretory granules, SG) and with

$6971 \mu \mathrm{M}$ SiR-tubulin (microtubules, MT) for $1 \mathrm{~h}$. Imaging was done with the DeltaVision

698 OMX (GE) microscope using an Olympus Plan Apochromat 60x/1.43 objective, yielding

699 dual channel images. Time-lapse movies were acquired in wide-field mode with $50 \mathrm{~ms}$

700 exposure-time and 10\% fluorescence intensity for each channel resulting in a final

701 speed of 2 frames per second (fps). Deconvolution was done with the SoftWorkx

702 software package running on-board the OMX. We created synthetic ground- truth

703 images of tubular networks and granular structures by simulate two-dimensional

704 trajectories and granular points on a pixel grid, respecting the known physical

705 properties (e.g. MT width and persistence length). We generated the corresponding

706 synthetic widefield input images by adding low-frequency Perlin noise mimicking auto-

707 fluorescence, convolving the result with the theoretical PSF of the microscope, and

708 adding Poisson and Gaussian noise mimicking camera noise. In total, we created around

7098000 synthetic patch-pairs of size $128 \times 128$. For both SG and MT, we trained a 2D

710 network (as before) to invert this degradation process and applied it on the respective

711 channel of the widefield images (Supp. Figure 13). More details can be found in Supp.

712 Notes 2.5.1. 
713 HeLa cell microtubule restoration and error map calculation. HeLa cells stably

714 expressing H2B-mCherry/mEGFP- $\alpha$-tubulin [77] were grown in DMEM containing 10\%

$715 \mathrm{FBS}, 100 \mathrm{U} / \mathrm{ml}$ penicillin and $100 \mathrm{mg} / \mathrm{ml}$ streptomycin at $37^{\circ} \mathrm{C}$ with $5 \% \mathrm{CO}_{2}$ in a

716 humidified incubator. Before imaging cells were seeded onto \#1.5 glass bottom $35 \mathrm{~mm}$

$717 \mathrm{u}$-Dish. Imaging was performed on a Zeiss Elyra PS.1 inverted microscope at $37^{\circ} \mathrm{C}$ and

$7185 \% \mathrm{CO}_{2}$ in TIRF mode with a Plan-Apochromat $100 \mathrm{x} / 1.46$ oil immersion objective

719 (Zeiss) and additional 1.6x magnification with 488nm laser illumination at an on-

720 sample intensity of $<10 \mathrm{~W} / \mathrm{cm}^{2}$. We created synthetic microtubule training data as

721 described before, resulting in around 5000 patch-pairs of size $128 \times 128$, and trained a

722 2D network as described before. Super-resolution images were reconstructed via SRRF

723 [78]. Error maps for both SRRF and CARE restoration were computed with SQUIRREL [79]

724 against the widefield reference frames.

725 Reliability measures and calibration. To model the inherent (aleatoric) uncertainty

726 of intensity predictions we adapted the final layers of the network to output a custom

727 probability distribution for every pixel of the restored image, instead of just a scalar

728 value. Specifically, the network predicted the parameters $\mu$ and $\sigma$ of a Laplace

729 distribution $p(z ; \mu, \sigma)=\frac{1}{2 \sigma} \exp (|z-\mu| / \sigma)$ for intensity value $z$. To represent the

730 (epistemic) model uncertainty for a specific experiment we trained an ensemble of $M$

731 networks (e.g. $\mathrm{M}=5$ ) and averaged their results (as a mixture model, $c f$. [80]). We

732 validated our probabilistic approach by adapting the concept of a calibrated classifier

733 [81] to the case of regression, which allows to compute accuracy/confidence curves and

734 to define an expected calibration error of a regression model (cf. Supp. Notes 3.1.3).

735 Furthermore, we quantified the normalized per-pixel disagreement of a network

736 ensemble via the average Kullback-Leibler divergence between the individual network

737 distributions and the ensemble mixture distribution. This allowed us to highlight image

738 regions with elevated disagreement scores that may indicate unreliable network

739 predictions (e.g. for very challenging low-SNR input; cf. Figure 5 \& Supp. Figure 28). For

740 an extensive and detailed discussion including all derivations see Supp. Notes 3.

\section{References (Online Methods)}

742 [50] O. Ronneberger, P. Fischer, and T. Brox. "U-net: Convolutional networks for

743 biomedical image segmentation". In: International Conference on Medical Image

744 Computing and Computer-Assisted Intervention. 2015.

745 [51] F. Chollet et al. Keras. https://github.com/fchollet/keras. 2015.

746 [52] M. Abadi et al. "Tensorflow: a system for large-scale machine learning." In: OSDI.

747 Vol. 16. 2016,

748 [53] Z. Wang et al. "Image quality assessment: from error visibility to structural

749 similarity”. In: IEEE Transactions on Image Processing 13.4 (2004),

750 [54] T. Boothe et al. "A tunable refractive index matching medium for live imaging cells, 751 tissues and model organisms". In: eLife 6 (2017). 
752 [55] C. Tomasi and R. Manduchi. "Bilateral filtering for gray and color images". In:

753 Computer Vision, 1998. Sixth International Conference on. IEEE. 1998,

754 [56] A. Buades, B. Coll, and J.-M. Morel. "A non-local algorithm for image denoising". In:

755 IEEE Conference on Computer Vision and Pattern Recognition. Vol. 2. 2005,

756 [57] A. Chambolle. "An algorithm for total variation minimization and applications". In:

757 Journal of Mathematical imaging and vision 20.1-2 (2004),

758 [58] K. Dabov et al. "BM3D image denoising with shape-adaptive principal component

759 analysis". In: SPARS'09-Signal Processing with Adaptive Sparse Structured

760 Representations. 2009.

761 [59] M. Maggioni et al. "Nonlocal transform-domain filter for volumetric data denoising 762 and reconstruction”. In: IEEE transactions on image processing 22.1 (2013),

763 [60] A. F. Sarrazin, A. D. Peel, and M. Averof. "A segmentation clock with two-segment 764 period-icity in insects". In: Science 336.6079 (2012),

765 [61] S. J. Brown et al. "The Red Flour Beetle, Tribolium castaneum (Coleoptera): A

766 Model for Studies of Development and Pest Biology". In: Cold Spring Harbor Protocols

7672009.8 (Aug. 2009),

768 [62] C. Sommer et al. "Ilastik: Interactive learning and segmentation toolkit". In:

769 Biomedical Imaging: From Nano to Macro, 2011 IEEE International Symposium on. IEEE. 770 2011,

771 [63] E. Jones, T. Oliphant, P. Peterson, et al. SciPy: Open source scientific tools for Python. 772 http://www.scipy.org. 2001.

773 [64] M. Mas`ka et al. "A benchmark for comparison of cell tracking algorithms". In:

774 Bioinformatics 30.11 (2014),

775 [65] A.-K. Classen et al. "Imaging Drosophila pupal wing morphogenesis". In:

776 Drosophila: Methods and Protocols (2008),

777 [66] C. Blasse et al. "PreMosa: extracting 2D surfaces from 3D microscopy mosaics". In:

778 Bioinformatics (2017),

779 [67] A. Shihavuddin et al. "Smooth 2D manifold extraction from 3D image stack". In:

780 Nature Communications 8 (2017).

781 [68] K. Li et al. "Optimal surface segmentation in volumetric images-a graph-theoretic 782 approach". In: IEEE transactions on pattern analysis and machine intelligence 28.1

783 (2006),

784 [69] X. Wu and D. Z. Chen. "Optimal net surface problems with applications". In:

785 International Colloquium on Automata, Languages, and Programming. Springer. 2002, 
[70] I. Arganda-Carreras et al. "Trainable Weka Segmentation: a machine learning tool

788 [71] J. Schindelin et al. "Fiji: an open-source platform for biological-image analysis". In:

789 Nature Methods 9.7 (July 2012),

790 [72] B. Aigouy, D. Umetsu, and S. Eaton. "Segmentation and Quantitative Analysis of

791 Epithelial Tissues". In: Drosophila: Methods and Protocols. Ed. by C. Dahmann. New York,

792 NY: Springer New York, 2016,

793 [73] L. A. Royer et al. "Adaptive light-sheet microscopy for long-term, high-resolution

794 imaging in living organisms”. In: Nature biotechnology 34.12 (2016),

795 [74] M. Weigert et al. "Isotropic Reconstruction of 3D Fluorescence Microscopy Images

796 Using Convolutional Neural Networks". In: International Conference on Medical Image

797 Computing and Computer-Assisted Intervention. 2017.

798 [75] M.-T. Ke, S. Fujimoto, and T. Imai. "SeeDB: a simple and morphology-preserving 799 optical clearing agent for neuronal circuit reconstruction". In: Nature neuroscience 16.8 800 (2013),

801 [76] A. Ivanova et al. "Age-dependent labeling and imaging of insulin secretory 802 granules”. In: Diabetes 62.11 (2013),

803 [77] N. Mchedlishvili et al. "Kinetochores accelerate centrosome separation to ensure 804 faithful chromosome segregation". In: J Cell Sci 125.4 (2012),

805 [78] N. Gustafsson et al. "Fast live-cell conventional fluorophore nanoscopy with Image 806 through super-resolution radial fluctuations". In: Nature Communications 7 (2016).

807 [79] S. Culley et al. "Quantitative mapping and minimization of super-resolution optical 808 imaging artifacts”. In: Nature methods 15.4 (2018),

809 [80] B. Lakshminarayanan, A. Pritzel, and C. Blundell. "Simple and Scalable Predictive 810 Uncertainty Estimation using Deep Ensembles". In: Advances in Neural Information 811 Processing Systems 30 (NIPS). 2017.

812 [81] C. Guo et al. "On Calibration of Modern Neural Networks". In: 34th International

813 Conference on Machine Learning (ICML). 2017. 


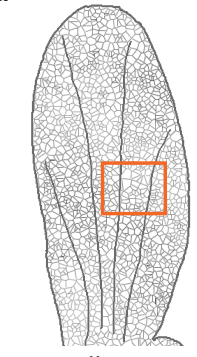

Full wing
Low SNR (Input)

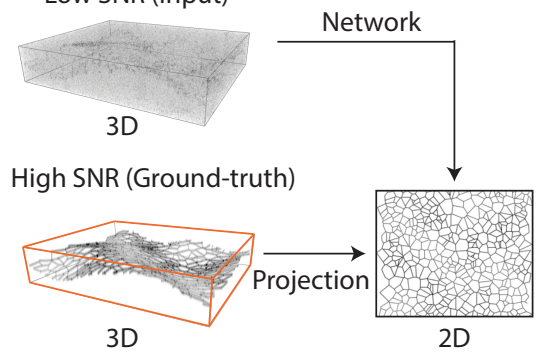

Network architecture

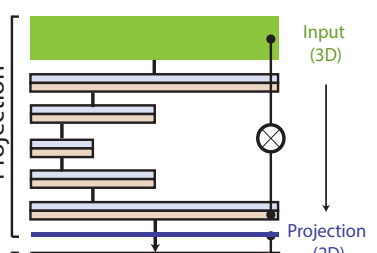

.

d

Reconstruction accuracy

MIP Network Premosa + NLM

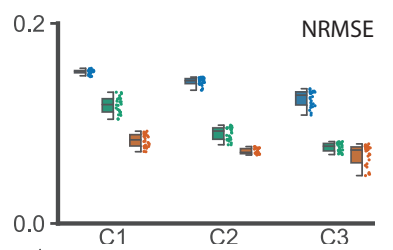

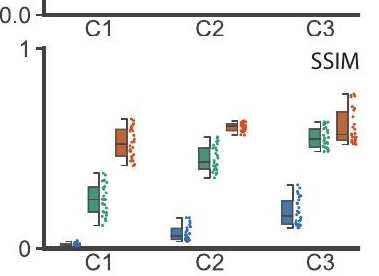

C

Input (Max Projection)

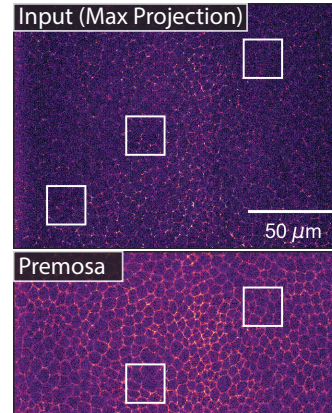

$\square$

Network
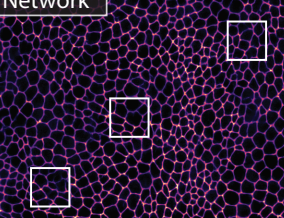

Ground-truth

War 200

D.

8
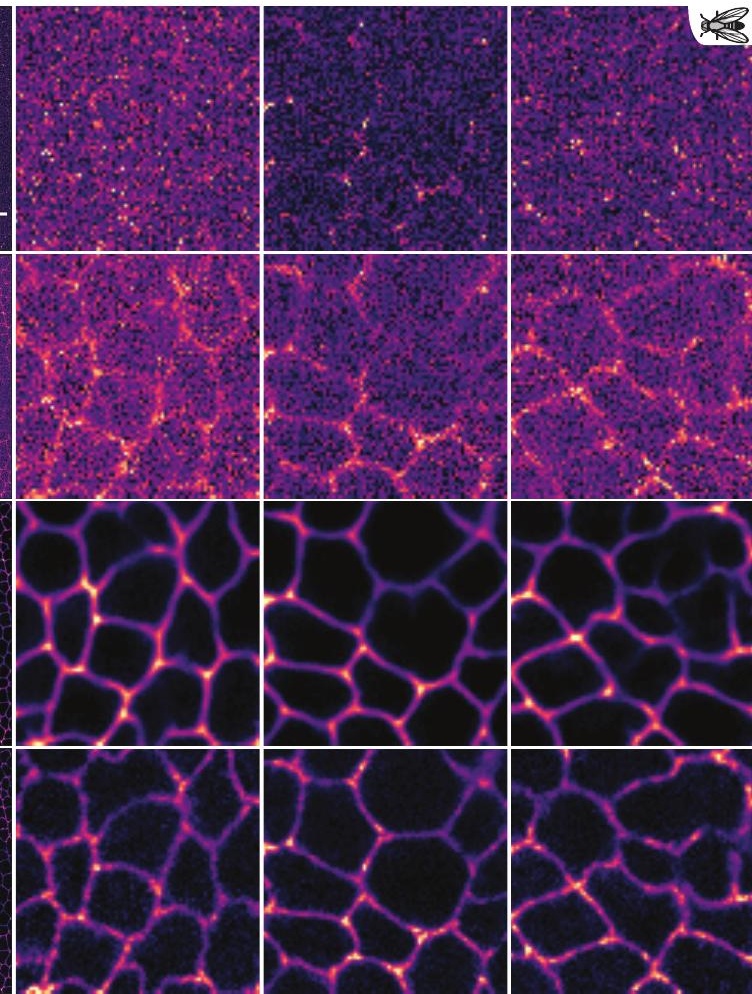


\section{a}

Training Data

Synthetic
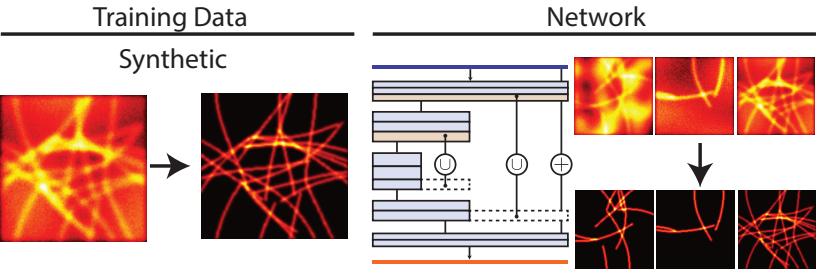

b
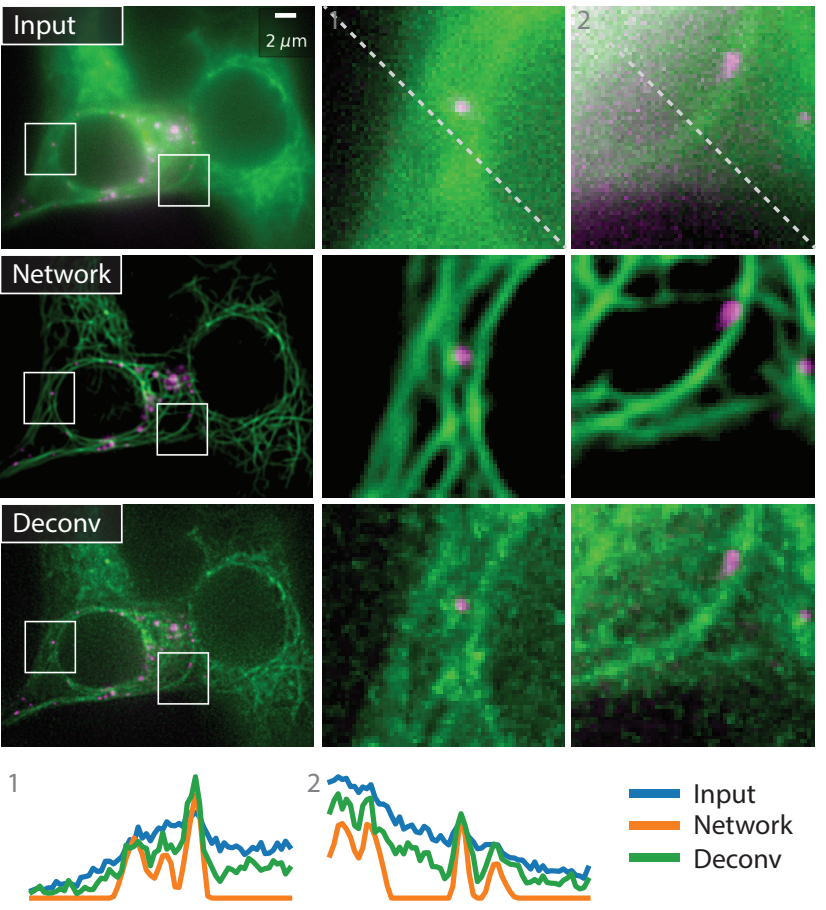

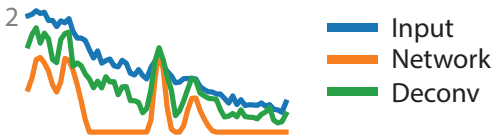

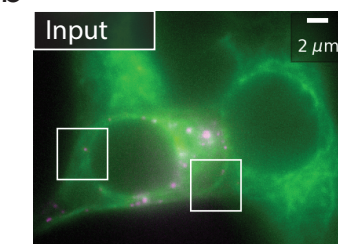

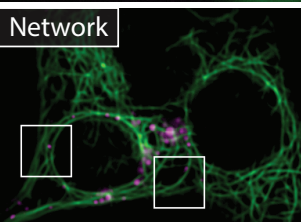

$$
\begin{aligned}
& \mathrm{N} \\
& \mathrm{D} \\
& \mathrm{D} \\
& 1
\end{aligned}
$$

Network

SRRF

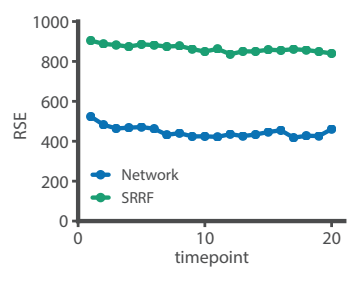

C
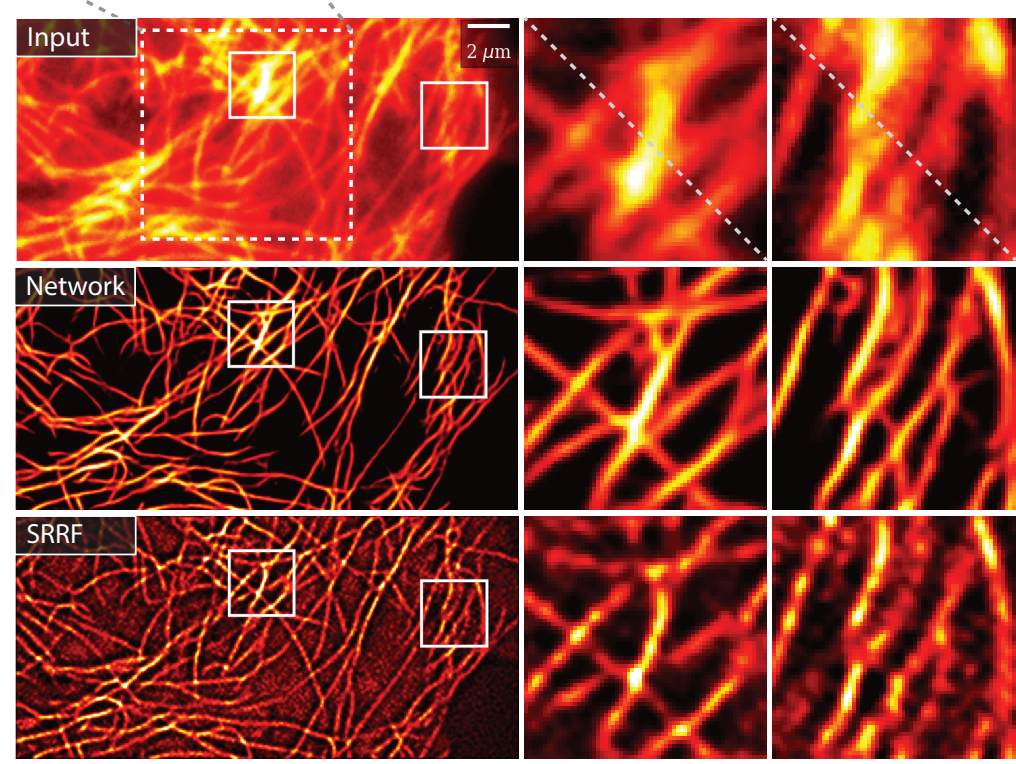

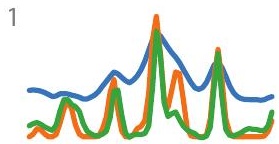

可
Input

- Network

SRRF 


\section{Predicting pixel-wise distributions}

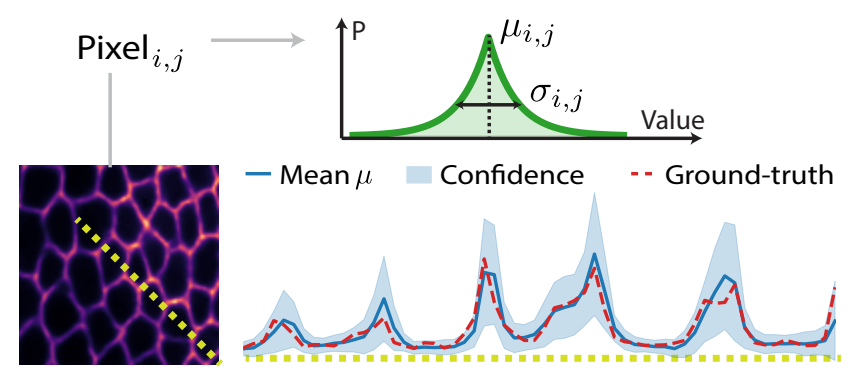

\section{Network Ensemble}

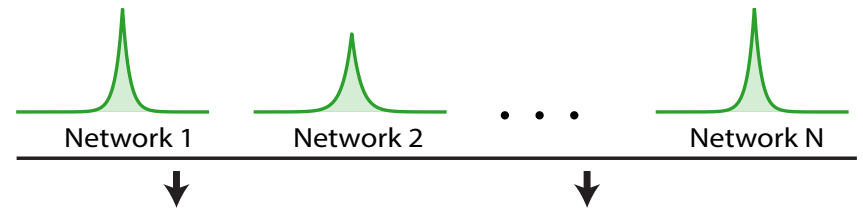

Ensemble Distribution Ensemble Disagreement $\mathcal{D}$

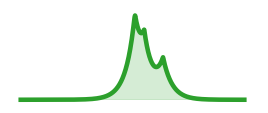

$\mathcal{D}=0 \quad$ All Distributions equal

$\mathcal{D}=1 \quad$ All Distributions max. different
Input

(MIP)
Network Ensemble

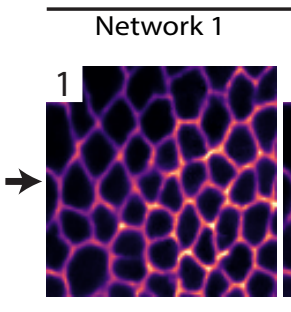
Network 2

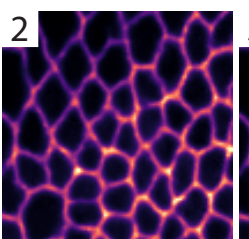

Network 3

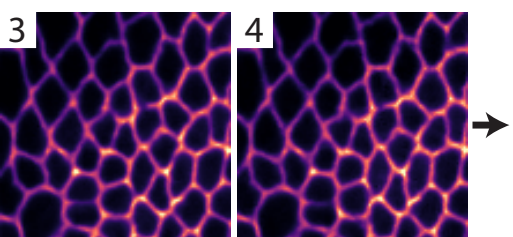

Ensemble Mean

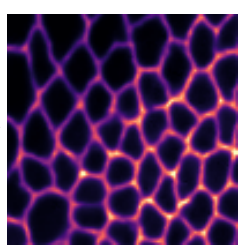

Ensemble Disagreement
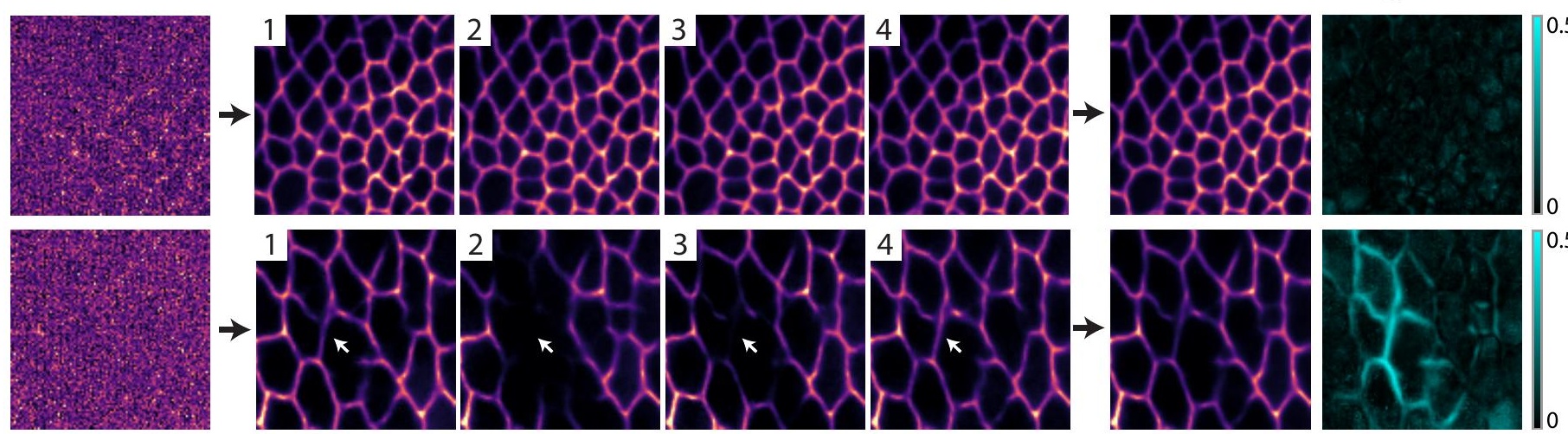